\title{
Relaciones interpersonales afectivas y desempeño laboral según variables sociodemográficas de los trabajadores de radio y televisión de Lima Metropolitana
}

\author{
Affective interpersonal relationships and work performance according \\ to sociodemographic variables of radio and television workers in \\ Metropolitan Lima
}

\author{
Manuel Bejarano Paredes ${ }^{1}$ \\ Radio Nacional del Perú
}

Recibido: $17-10-19$

Aceptado: $17-12-19$

\section{Resumen}

La presente investigación estudia las diferencias entre las relaciones interpersonales y el desempeño laboral según variables sociodemográficas en trabajadores de tres empresas estatales de radio y televisión en Lima Metropolitana, la muestra estuvo constituida por 90 trabajadores (44\% varones y 56\% mujeres). Para la recolección de datos se utilizó el Cuestionario de Conductas de Desempeño Laboral y la Escala de Relaciones Interpersonales Afectivas en el Trabajo (SRIT), se identificó a través del estadístico de Kolmogorov-Smirnov que los datos recogidos presentaban características no paramétricas por lo que el análisis de resultados se hizo mediante el estadístico Rho de Spearman d U de Man Whitney y el estadístico Kruskal-Wallis. Los resultados indicaron que no existe una correlación significativa entre las entre las relaciones interpersonales afectivas y el desempeño laboral, un análisis más minucioso de sus componentes arroja una relación moderada entre ellas. Asimismo, la comparación entre las relaciones interpersonales afectivas y el desempeño laboral, con las variables sociodemográficas no mostraron relación significativa.

Palabras clave: Relaciones afectivas; desempeño laboral; trabajadores de radio y televisión; Perú.

\begin{abstract}
The present investigation studies interpersonal relationships and work performance in workers of three state-owned radio and television companies in Metropolitan Lima, the sample consisted of 90 workers ( $44 \%$ male and $56 \%$ female). For the data collection, the Labor Performance Behavior Questionnaire and the Scale of Affective Interpersonal Relationships at Work (SRIT) were used, it was identified through the Kolmogorov-Smirnov statistic that the data collected presented non-parametric characteristics so that Analysis of results was done using the Rho
\end{abstract}

\footnotetext{
1 Psicólogo profesional, Periodista en Radio Nacional del Perú. E-mail: manrebe@hotmail.com

(C) Los autores. Este artículo es publicado por la Revista de Investigación en Psicología de la Facultad de Psicología, Universidad Nacional Mayor de San Marcos. Este es un artículo de acceso abierto, distribuido bajo los términos de la licencia Creative Commons Atribucion - No Comercia_Compartir Igual 4.0 Internacional. (http://creativecommons.org/licenses/by-nc-sa/4.0/) que permite el uso no comercial, distribución y reproducción en cualquier medio, siempre que la obra original sea debidamente citada.
} 
statistic of Spearman d U of Man Whitney and the statistician Kruskal-Wallis. The results indicated that there is no significant correlation between the interpersonal affective relationships and work performance, a more thorough analysis of its components yields a moderate relationship between them. Likewise, the comparison between affective interpersonal relationships and work performance, with sociodemographic variables did not show a significant relationship.

Keywords: affective relations; radio workers; Tv workers; job performance; Peru.

En nuestro este mundo globalizado se viene priorizando cada vez más el capital humano y su bienestar en las organizaciones, esto debido a que el rol de los trabajadores es fundamental para una empresa pues representan la imagen de esta y son la mano de obra que creará el producto de la organización (Montoya y Boyero, 2015). Es importante resaltar que en las organizaciones la creación de productos dependerá en gran medida del desempeño laboral, que es entendida como algo fundamental y primordial para rendir en las tareas y alcanzar los objetivos y metas a partir de personal productivo (Halbesleben y Wheeler, 2008)

Los colaboradores de todas las empresas evocan un desempeño laboral en función de las características particulares del lugar de trabajo. Esto involucrará ser competente laboralmente, expresando de manera eficiente los, conocimientos, aptitudes, valores y deseos, actitudes, motivaciones pertinentes a las exigencias de la empresa (Juaneda y Gonzáles, 2007), por lo cual en la actualidad el desempeño laboral se concibe como un proceso complejo en el que están involucrados diversos actores en una organización (Swanson y Holton,2001).El desempeño organizacional, por su parte, incluirá los conductas comprometidas con, entre las que destacan la fidelidad, la responsabilidad y el ser leal (Coleman \& Borman, 2000) y estará caracterizado por evidenciar a un trabajador con habilidades para adaptarse a diversos ambientes, comunicarse de manera efectiva de manera grupal e individual, poseer la intención de generar impacto de manera dinámica en los acontecimientos para lograr un propósito y la continua actualización de conocimientos técnicos / profesionales (Lévy-Leboyer,1997). Este es influido por muchos factores externos e internos a la organización, uno de ellos es la relación interpersonal que se da en el ambiente laboral tanto entre los trabajadores de un mismo nivel jerárquico como en los miembros jefes y subordinados.

Entendiendo que los espacios laborales en su mayoría van a implicar establecer relaciones sociales, la calidad de las relaciones interpersonales en el contexto laboral puede generar un conjunto de reacciones en las personas provocando situaciones de estrés que terminan afectando el desempeño laboral y, por tanto, la productividad; además, las relaciones conflictivas o poco colaborativas podrían llegar al favoritismo y la falta de objetividad, crear suspicacias, tensiones y deterioro del ambiente, esto hace que, en muchas ocasiones, una de las partes opte por acabar dejando su puesto laboral para incorporarse a otra compañía (Chiavenato, 2002). Existen diferentes posturas de cómo entender las relaciones interpersonales 
en el ámbito laboral, entendiendo que en este sistema existe una complejidad de interacciones entre empleado-empleador, grupo de pares, relaciones con personas de mayor y menor jerarquía, cadenas de mando y relaciones afectivo-amorosas; aunado al estrés que muchas veces se percibe en una actividad laboral, por lo que es un ambiente sumamente complejo de interacción. Por lo cual Yañez et al. (2010) sostienen que existen dos tipos de relaciones principales dentro del ambiente laboral; las relaciones interpersonales entre pares y las relaciones interpersonales con los jefes (Yáñez, 2006; Yáñez, Loyola y Huenumilla, 2009).

Entendiendo las relaciones interpersonales entre pares, como la interacción entre dos personas que laboran dentro de la misma organización y tienen un nivel jerárquico equivalente y mantienen una relación amical, no siendo necesariamente tener características similares en el salario, horario, área, etc., se viene identificando que las relaciones interpersonales afectivas, pueden mejorar el desempeño laborar y aumentar el rendimiento, contrariamente, en el sentido negativo, pueden ocasionar descontento, insatisfacción, problemas laborales e incluso reducción de la productividad, más aún, cuando dichas relaciones alteran otras variables, como el clima laboral, mediante "chismes", "rumores" y otras formas de comunicación que pueda generar conflictos (Chiaburu y Harrison ,2008;Yañez, Arenas y Ripoll 2010).

Por lo que es entendible que el papel de las relaciones interpersonales juegue un rol fundamental en la organización ya que influye en el clima y la cultura organizacional y en las estrategias de producción (Chiaburu y Harrison ,2008), por lo que es de suma importancia conocer el estado actual de las relaciones interpersonales e identificar situaciones de riesgo para generar programas/ intervenciones que mejoren estas relaciones, tanto de forma individual como de grupos. El desempeño laboral, por su parte, es de vital interés para los directivos de la organización, en tanto y en cuanto existan esfuerzos de la organización, para optimizar la producción, por lo que se busca desarrollar diferentes estrategias para aumentar la productividad, mejorado el clima laboral, las relaciones interpersonales y la satisfacción laboral, identificándose nuevamente a las relaciones interpersonales, como un factor sumamente importante para la organización.

Es por ello que, ante problemas de relaciones interpersonales insatisfactorias y/o bajo desempeño laboral es necesario tomar medidas/intervenciones centradas en los trabajadores (Yáñez, 2006). Ya que ambos son problemas muy fuertes que pueden afectar otros estratos o áreas organizacionales, esto es particularmente importante en organizaciones que gestionan y difunden información, como es el caso de las empresas periodísticas o de telecomunicaciones, ya que la información que difunden impacta en la vida diaria de toda una nación.

En ese sentido, en los medios de comunicación de radio y televisión de Lima Metropolitana, como en cualquier organización de esta naturaleza, existe este tipo 
de circunstancias que merece nuestra atención. Debido a esto, el presente estudió se propuso investigar las diferencias entre el desempeño laboral y las relaciones interpersonales afectivas según el número de hijos, tipo de contrato, sexo, cargo; a fin de despejar interrogantes sobre la asociación de las relaciones interpersonales afectivas con el desempeño laboral

\section{MÉTODO}

El estudio es de tipo cuantitativo no experimental, de diseño descriptivocomparativo pues está orientada a determinar la relación o grado de asociación existente entre dos o más variables de interés en una muestra (Sánchez y Reyes 2006; Hernández, Fernández y Baptista, 2010), en este caso se buscará conocer la relación existente entre el desempeño laboral y las relaciones interpersonales afectivas de 90 trabajadores de Radio La Crónica, Radio Nacional y TV Perú, además el estudio está orientado a determinar algunos factores descriptivos porque analiza las características de los participantes.

\section{Muestra}

Se ha contado con una población de trabajadores mayores de edad, productores, periodistas, secretarias y camarógrafos de Radio La Crónica, Radio Nacional y TV Perú. La selección de la muestra se determinó aplicándose un muestreo no probabilístico circunstancial (Hernández et al., 2010) y para lo cual se consideró ciertos criterios para su inclusión en la investigación: Todos los trabajadores entre los 18 y 70 años de edad, trabajadores con más de 3 meses de antigüedad en la empresa y trabajadores que deseen participar de manera voluntaria del estudio; y se excluyó a: personal menor a 18 años y mayor de 70 años, trabajadores con antigüedad menor a 3 meses y personas que no acepten de manera voluntaria participar en el estudio.

La muestra quedó establecida con 90 trabajadores y se identificó que los participantes hombres $(n=40)$ representan el $44.4 \%$ de la muestra y las mujeres $(\mathrm{n}=50)$ responden al 55.6\% de los evaluados para el estudio, además se identificó que el $91.1 \%$ son heterosexuales, en su mayoría se identifican los estados civiles de solteros $(\mathrm{n}=33,36.7 \%)$ y casados $(\mathrm{n}=38,42.2 \%)$ donde su grado de instrucción es de técnicos y profesionales, pertenecientes a las áreas de producción, prensa, operaciones, programación y técnica, así mismo se analizó otras características de la muestra.

\section{Variables de Estudio}

Las variables de estudio corresponden a los indicadores de la Escala de Satisfacción en las Relaciones Interpersonales en el trabajo (SRIT) de Yañez et a. 2010, compuesta por 2 dimensiones (Relación con el jefe y relación con los pares) y el Cuestionario de desempeño laboral de Flores y Rodríguez (2006). compuesta por 
3 dimensiones (Dimensión ciudadanía interpersonal, ciudadanía organizacional y dimensión de escrupulosidad laboral.

\section{Técnicas e instrumentos de recolección de datos}

Inicialmente se realizó las coordinaciones correspondientes con la oficina de relaciones humanas de cada empresa, solicitando los permisos correspondientes. Hemos encuestado y administrado dos instrumentos, los cuales fueron revisados para ser aplicados y ambos se encuentran adaptados y validados a población peruana. se utilizaron estrategias de evaluación colectiva, cuidando que los participantes estén en un ambiente bien iluminado, silencioso, cómodo y que facilite la aplicación de los instrumentos. Al finalizar las evaluaciones, antes de recibir las pruebas se constató que todas las preguntas estén adecuadamente marcadas, solicitando al participante que termine de llenar la prueba, si esta no estuviera completa.

\section{Instrumentos}

Escala de Satisfacción en las Relaciones Interpersonales en el Trabajo (SRIT) Elaborado por Yañez et al. (2010) en Chile. Este instrumento se basó en las investigaciones previas de Yáñez, 2006 y Yáñez, Loyola y Huenumilla, 2009.

En el estudio de sus propiedades métricas, se examinó la estructura interna del instrumento mediante análisis factorial exploratorio (Muñiz, 2002; Pohlmann, 2004; Costello \& Osborne, 2005) y se obtuvo un KMO de .93 lo que indicó una adecuación de datos posible para hacer el análisis factorial, mediante el método de extracción de Componentes Principales seguido por rotación Varimax y se evidenció que la mejor representación de los ítems se lograba con una estructura compuesta por dos factores.; el factor 1, asociado a percepción de satisfacción con las relaciones interpersonales con la jefatura y el valor propio y el factor 2 asociado a la percepción de satisfacción con las relaciones interpersonales con los pares en el trabajo y explican el $66.50 \%$ de la varianza. Al evaluar la confiabilidad a través de la consistencia interna con el alfa de Cronbach se obtuvo 0, 94. Los ítems presentaban una correlación ítems-test adecuados, entre 0,53 y 0,80 .

Para la presente investigación se realiza el análisis de las propiedades métricas en la muestra actual, inicialmente se realiza un análisis descriptivo de los puntajes obtenido de la SRIT, analizando los ítems (Tabla 1), todos los ítems superaron los valores de correlación ítem test mayor a .20 por lo que no se eliminó ninguno (Hogan, 2004). Para la confiabilidad se utilizó el estadístico del alfa de Crombach, en las dimensiones de Relación con el jefe y Relación con los pares, obteniendo niveles de confiabilidad aceptables $>.70$ (DeVillis, 2012). De igual forma se analizó la confiabilidad de toda la escala obteniendo índices adecuados de confiabilidad (Tabla 2) 


\section{Tabla 1}

Puntajes de tendencia central, dispersión, correlación item-test y a si el elemento se ha suprimido en la escala de relaciones interpersonal en el trabajo (SRIT)

\begin{tabular}{|c|c|c|c|c|c|}
\hline & & $M$ & $D E$ & Correlación ítem-test & $\alpha$ si el elemento se ha suprimido \\
\hline \multirow{6}{*}{ 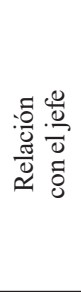 } & Ítem 1 & 2.68 & 1.140 & .742 & .865 \\
\hline & Ítem 2 & 2.61 & 1.067 & .765 & .862 \\
\hline & Ítem 3 & 2.72 & .995 & .674 & .876 \\
\hline & Ítem 4 & 2.83 & 1.052 & .664 & .878 \\
\hline & Ítem 5 & 2.64 & 1.164 & .688 & .875 \\
\hline & Ítem 6 & 2.87 & 1.062 & .719 & .869 \\
\hline \multirow{7}{*}{ 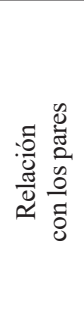 } & Ítem 7 & 2.79 & 1.033 & .308 & .838 \\
\hline & Ítem 8 & 3.20 & .997 & .580 & .794 \\
\hline & Ítem 9 & 3.08 & 1.052 & .599 & .790 \\
\hline & Ítem 10 & 3.13 & 1.030 & .729 & .767 \\
\hline & Ítem 11 & 2.99 & 1.117 & .571 & .796 \\
\hline & Ítem 12 & 3.08 & .915 & .583 & .794 \\
\hline & Ítem 13 & 3.17 & .963 & .593 & .792 \\
\hline
\end{tabular}

Tabla 2

Análisis de confiabilidad de las relaciones interpersonal afectivas en el trabajo (SRIT)

\begin{tabular}{llcccc} 
& & $\boldsymbol{M}$ & $\boldsymbol{D E}$ & $\mathbf{N}^{\circ}$ Ítems & A \\
\hline \multirow{2}{*}{$\begin{array}{l}\text { Relaciones } \\
\text { interpersonales }\end{array}$} & Relación con el jefe & 16.36 & 5.213 & 6 & .890 \\
& Relación con los pares & 21.43 & 4.94 & 7 & .821 \\
& TOTAL & 37.79 & 9.02 & 13 & .894 \\
\hline
\end{tabular}

\section{Cuestionario de Conductas de Desempeño Laboral de Flores y Rodríguez} (2006), creado en Venezuela con el objetivo de medir los niveles de desempeño contextual presentes en la muestra del estudio. Flores y Rodríguez desarrollaron el instrumento en base de la teoría de Coleman \& Borman (2000), compuesta por 24 ítems: ocho (8) para la dimensión ciudadanía interpersonal $(\alpha=.63)$, diez (10) para la dimensión ciudadanía organizacional $(\alpha=71)$, y seis (6) para la dimensión de escrupulosidad laboral $(\alpha=.51)$. Se utilizó una escala Likert de 5 puntos: (1) nunca, (2) casi nunca, (3) a veces, (4) casi siempre y (5) siempre.

Se realizó los análisis de fiabilidad en la muestra actual, para lo cual se realizó un análisis descriptivo de los puntajes obtenido del cuestionario de conductas de desempeño laboral, analizando los ítems (Tabla 3), todos los ítems superaron los valores de correlación ítem test mayor a .20 por lo que no se eliminó ninguno (Hogan, 2004). Para la confiabilidad se utilizó el estadístico del alfa de Crombach (Tabla 4), en las dimensiones de Interpersonal, Organizacional y Proactividad, obteniendo niveles de confiabilidad aceptables $>.70$ (De Vellis, 2012). 
Relaciones interpersonales afectivas y desempeño laboral según variables sociodemográficas de los trabajadores de radio y televisión de Lima Metropolitana

Tabla 3

Puntajes de promedio, desviación estándar, correlación item-test y a si el elemento se ha suprimido en el cuestionario de conductas de desempeño laboral

\begin{tabular}{|c|c|c|c|c|c|}
\hline & & $M$ & $D E$ & Correlación ítem-test & $\alpha$ si el elemento se ha suprimido \\
\hline \multirow{8}{*}{ 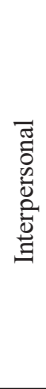 } & Ítem 4 & 4.10 & .654 & .540 & .779 \\
\hline & Ítem 5 & 4.09 & .729 & .614 & .767 \\
\hline & Ítem 8 & 4.13 & .782 & .512 & .781 \\
\hline & Ítem 12 & 3.78 & .897 & .570 & .772 \\
\hline & Ítem 17 & 3.76 & .739 & .631 & .764 \\
\hline & Ítem 19 & 3.69 & .956 & .477 & .790 \\
\hline & Ítem 22 & 3.90 & .688 & .450 & .790 \\
\hline & Ítem 23 & 3.71 & .782 & .370 & .802 \\
\hline \multirow{12}{*}{ 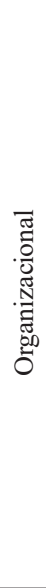 } & Ítem 6 & 3.97 & .678 & .607 & .842 \\
\hline & Ítem 7 & 4.01 & .727 & .584 & .843 \\
\hline & Ítem 9 & 4.33 & .734 & .679 & .837 \\
\hline & Ítem 10 & 3.82 & .943 & .528 & .847 \\
\hline & Ítem 11 & 3.67 & .793 & .320 & .860 \\
\hline & Ítem 13 & 4.08 & .824 & .740 & .831 \\
\hline & Ítem 14 & 4.08 & .707 & .645 & .839 \\
\hline & Ítem 15 & 3.99 & .786 & .603 & .841 \\
\hline & Ítem 16 & 4.00 & .764 & .699 & .835 \\
\hline & Ítem 21 & 4.06 & .709 & .629 & .840 \\
\hline & Ítem 25 & 3.84 & .778 & .181 & .869 \\
\hline & Ítem 26 & 3.53 & .962 & .325 & .864 \\
\hline \multirow{7}{*}{ 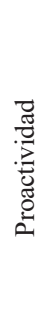 } & Ítem 1 & 4.21 & .786 & .641 & .724 \\
\hline & Ítem 2 & 4.14 & .728 & .575 & .739 \\
\hline & Ítem 3 & 4.06 & .770 & .535 & .746 \\
\hline & Ítem 18 & 4.08 & .851 & .596 & .733 \\
\hline & Ítem 20 & 4.27 & .614 & .467 & .761 \\
\hline & Ítem 24 & 3.74 & .868 & .253 & .808 \\
\hline & Ítem 27 & 4.22 & .614 & .538 & .750 \\
\hline
\end{tabular}

Tabla 4

Análisis de confiabilidad de la escala Relación en el cuestionario de conductas de desempeño laboral

\begin{tabular}{llcccc}
\hline & & $\boldsymbol{M}$ & $\boldsymbol{D E}$ & $\mathbf{N}^{\circ}$ Ítems & $\mathbf{A}$ \\
\hline \multirow{5}{*}{ Desempeño laboral } & Interpersonal & 31.16 & 4.07 & 8 & .803 \\
& Organizacional & 47.38 & 5.90 & 12 & .857 \\
& Proactividad & 28.72 & 3.46 & 7 & .780 \\
\cline { 2 - 6 } & TOTAL & 99.81 & 11.27 & 27 & .929 \\
\hline
\end{tabular}




\section{RESULTADOS}

\section{Resultados Descriptivos de las variables de estudio}

Se analizó las puntuaciones de las dimensiones de las relaciones interpersonales y el desempeño laboral y se identificó los siguientes resultados:

Las puntuaciones que arrojaron los datos sobre las relaciones interpersonales en los trabajadores indican que existe una tendencia de percepción promedio (41.1\%) sobre sus relaciones, el $32.2 \%$ puntúa en el nivel alto y finalmente solo el $26.7 \%$ arroja un nivel bajo en sus relaciones. En lo relacionado a las dimensiones de la variable de estudio, se identificó que los participantes de la muestra describen la "relación con su jefe" en su mayoría a nivel promedio pues representan el 41.1\%, seguido del $31.1 \%$ quienes describen una baja relación y solo el $27.8 \%$ en el nivel la describe como bueno; probablemente se debe a que las relaciones con sus jefes no son óptimas como se esperaría. Por otra parte, en la dimensión "relación con los pares" la mayoría de los colaboradores reportan un nivel normal $41.1 \%$ de vinculo con sus compañeros, el 33\% lo describe como inadecuadas relaciones y sólo el $27.8 \%$ las identifica como buenas relaciones.

Tabla 5

Descripción del nivel de relaciones interpersonales y sus dimensiones

\begin{tabular}{|c|c|c|c|c|c|}
\hline & & Niveles & Frecuencia & $\%$ valido & $\%$ acumulado \\
\hline \multirow{3}{*}{$\begin{array}{l}\text { Relaciones interpersonales } \\
\text { afectivas }\end{array}$} & Bajo & 15 a 23 & 24 & 26.7 & 33.3 \\
\hline & Medio & 33 a 43 & 37 & 41.1 & 67.8 \\
\hline & Alto & 44 a 56 & 29 & 32.2 & 100 \\
\hline \multirow{3}{*}{ Relación con el jefe } & Bajo & 06 a 13 & 30 & 31.1 & 31.1 \\
\hline & Medio & 14 a 20 & 37 & 41.1 & 72.2 \\
\hline & Alto & 21 a 27 & 23 & 27.8 & 100 \\
\hline \multirow{3}{*}{ Relación con los pares } & Bajo & 08 a 19 & 30 & 33.3 & 33.3 \\
\hline & Medio & 20 a 25 & 37 & 41.1 & 74.4 \\
\hline & Alto & 26 a 31 & 23 & 27.8 & 100 \\
\hline
\end{tabular}

Por otro lado, en lo vinculado con la variable Desempeño Laboral (Tabla 6) los participantes de la muestra; se encuentran en el nivel promedio en un $38.9 \%$, seguido del $28.9 \%$ en el nivel bajo y el $32.2 \%$ en el nivel bueno, considerando estos hallazgos se sugiere una productividad eficiente de los empleados. En lo vinculado con las dimensiones del constructo se evidenció que en la "dimensión interpersonal" un $42.2 \%$ se encuentra en un nivel promedio, seguido del $30 \%$ en el nivel malo (Bajo) y solo el $27.8 \%$ en el nivel bueno (Alto), en este sentido, estos hallazgos sugieren que la destreza interpersonal resulta prevalente en la muestra; por otro lado, en la "dimensión organizacional" un 33,4\% se encuentra en un nivel promedio, seguido del 33,3\% en el nivel malo (Bajo) y de igual manera el 
33,3\% en el nivel bueno (Alto) y se señala entonces, que la manifestación sobre la organización de la empresa resulta equivalente. Finalmente, en el caso de los niveles de la "dimensión proactividad" en la escala de desempeño laboral, donde un 34,4\% se encuentra en un nivel promedio, seguido del $34,4 \%$ en el nivel malo (Bajo) y el $31,2 \%$ en el nivel bueno (Alto), estos hallazgos sugieren que la proactividad en el centro de trabajo resulta una cualidad regular en la organización.

Tabla 6

Niveles de la dimensión interpersonal de la escala de desempeño laboral

\begin{tabular}{lllccc}
\hline \multirow{5}{*}{ Desempeño Laboral } & & Niveles & Frecuencia & \% valido & \% acumulado \\
\cline { 2 - 6 } & Bajo & 69 a 95 & 26 & 28.9 & 28.9 \\
\cline { 2 - 6 } & Medio & 96 a 106 & 35 & 38.9 & 67.8 \\
\cline { 2 - 6 } Destreza Interpersonal & Alto & 107 a 122 & 29 & 32.2 & 100 \\
\hline \multirow{5}{*}{ Organizacional } & Bajo & 12 a 22 & 27 & 30 & 30 \\
& Medio & 23 a 25 & 38 & 42.2 & 72.2 \\
& Alto & 26 a 30 & 25 & 27.8 & 100 \\
\hline \multirow{5}{*}{ Proactividad } & Bajo & 30 a 45 & 30 & 33.3 & 33.3 \\
& Medio & 46 a 50 & 30 & 33.4 & 66.7 \\
& Alto & 51 a 59 & 30 & 33.3 & 100 \\
\hline & Bajo & 18 a 27 & 31 & 34.4 & 34.4 \\
& Medio & 28 a 30 & 31 & 34.4 & 68.9 \\
\hline
\end{tabular}

\section{Análisis comparativo}

Inicialmente, se realizó un análisis de normalidad para identificar el tipo de distribución de los datos a través de la prueba de Kolmogorov-Smirnov con la corrección de la significación de Lilliefors y se identificó que presenta una distribución no paramétrica para las relaciones interpersonales como para el desempeño laboral, ya que los valores son significativos, rechazando la hipótesis nula para la escala de relaciones interpersonales.

\section{Según número de hijos}

Se analizó la diferencia de grupos mediante el estadístico Kruskal-Wallis y se identificó que en lo relacionado con la variable relaciones interpersonales existen diferencias estadísticamente significativas según el número de hijos de los trabajadores de radio y televisión en Lima Metropolitana, encontrando que existen diferencias estadísticamente significativas el nivel de Relación con el jefe y la Relación con los pares; es decir que las personas que tienen solo un hijo tienen mayores puntajes de relaciones con el jefe que los que tienen tres a más hijos. 
Sin embargo, con respecto a si el número de hijos alteraba las puntuaciones sobre el desempeño laboral no se identificó resultados estadísticamente significativos, en este sentido y con base en los hallazgos se mantiene la Hipótesis Nula (H0) y se suspende la Hipótesis del estudio; por ello se afirma que no existen diferencias estadísticamente significativas en el desempeño laboral según el número de hijos de los trabajadores de radio y televisión en Lima Metropolitana.

Tabla 7

Diferencia de medias entre las variables de estudio según el número de hijos

\begin{tabular}{lll}
\hline \multicolumn{1}{c}{ Variable } & \multicolumn{1}{c}{ Dimensión } & P \\
\hline \multirow{2}{*}{ Relaciones interpersonales } & Relación con el jefe & .001 \\
& Relación con los pares & .005 \\
\hline \multirow{3}{*}{ Desempeño laboral } & Interpersonal & .656 \\
& Organizacional & .353 \\
& Proactividad & .472 \\
\hline
\end{tabular}

\section{Según tipo de contrato}

Se analizó la variable Relaciones interpersonales según el tipo de contrato que poseen los trabajadores de Radio y Televisión en Lima Metropolitana (Tabla 8) y se identificó que no existe diferencias estadísticamente significativas en las relaciones interpersonales afectivas según el tipo de contrato. Sin embargo, en lo vinculado al desempeño laboral se observó que si existen diferencias en la dimensión productividad según el tipo de contrato (CAS, plazo determinado y plazo indeterminado) que tenga el empleado.

Tabla 8

Diferencia de medias entre las variables según el tipo de contrato

\begin{tabular}{lll}
\hline \multicolumn{1}{c}{ Variable } & \multicolumn{1}{c}{ Dimensión } & P \\
\hline \multirow{2}{*}{ Relaciones interpersonales } & Relación con el jefe & .248 \\
& Relación con los pares & .767 \\
\hline \multirow{3}{*}{ Desempeño laboral } & Interpersonal & .062 \\
& Organizacional & .085 \\
& Proactividad & $\mathbf{. 0 2 1}$ \\
\hline
\end{tabular}

\section{Según condición laboral}

En lo vinculado con la relación entre las variables de estudio y la condición laboral de los trabajadores, se halló que no existe relación estadísticamente significativa entre las dimensiones interpersonales y su condición de trabajo (Tabla 9); asimismo, los hallazgos no muestran estadísticas significativas en la comparación 
de las dimensiones del desempeño. En este sentido y con base en los hallazgos se mantiene la Hipótesis Nula (H0) y se suspende la Hipótesis del estudio.

\section{Tabla 9}

Diferencia de medias entre las relaciones interpersonales afectivas y el desempeño laboral según la condición laboral

\begin{tabular}{llccc}
\hline Variable & Dimensiones & U de Mann-Whitney & Z & p \\
\hline $\begin{array}{l}\text { Relaciones } \\
\text { interpersonales }\end{array}$ & Relación con el jefe & 532,000 & $-1,173$ &, 241 \\
& Relación con los pares & 493,000 & $-1,567$ &, 117 \\
\hline \multirow{2}{*}{$\begin{array}{l}\text { Desempeño } \\
\text { laboral }\end{array}$} & Interpersonal & 482,000 & $-1,681$ &, 093 \\
& Organizacional & 595,500 &,- 531 &, 595 \\
\hline
\end{tabular}

\section{Según nivel jerárquico}

En la tabla 10, se puede observar el análisis comparativo entre las relaciones interpersonales y el nivel jerárquico de los empleados de Radio y Televisión en Lima Metropolitana. Según el análisis realizado no se identifican relaciones estadísticas significativas en la comparación de las dimensiones de las relaciones interpersonales afectivas. De la misma manera con las dimensiones de la variable Desempeño laboral, se mantiene la Hipótesis Nula (H0) y se precisa que no existen diferencias estadísticamente significativas en el desempeño laboral según el nivel jerárquico de los trabajadores de radio y televisión en Lima Metropolitana.

\section{Tabla 10}

Diferencia de medias entre las relaciones interpersonales afectivas y el desempeño laboral según la nivel jerárquico

\begin{tabular}{llccc}
\hline Variable & Dimensiones & U de Mann-Whitney & Z & p \\
\hline \multirow{2}{*}{ Relaciones } & Relación con el jefe & 127,500 &,- 034 &, 973 \\
interpersonales & Relación con los pares & 71,000 & $-1,322$ &, 186 \\
\hline \multirow{3}{*}{ Desempeño laboral } & Interpersonal & 482,000 & $-1,681$ &, 093 \\
& Organizacional & 595,500 &,- 531 &, 595 \\
& Proactividad & 633,000 &,- 153 &, 879 \\
\hline
\end{tabular}

\section{Relaciones según otras variables sociodemográficas}

En la tabla 11 se evidencia el muestra el análisis comparativo entre Relaciones interpersonales afectivas, el desempeño laboral y un grupo de variables sociodemográficas como orientación sexual, Estado Civil, Grado de Instrucción, Empresa a la que pertenecen, Puesto, Ingreso salarial, Edad, Personas dependientes, Área laboral y Tiempo de servició. Como se observa, no se hallaron diferencias estadísticamente significativas en ningún caso. 
Tabla 11

Relación entre los constructos de estudio y otras variables sociodemográficas

\begin{tabular}{lccccc}
\hline & \multicolumn{2}{c}{ Relaciones interpersonales } & \multicolumn{3}{c}{ Desempeño laboral } \\
\hline & $\begin{array}{c}\text { Relación con } \\
\text { el jefe }\end{array}$ & $\begin{array}{c}\text { Relación con } \\
\text { los pares }\end{array}$ & Interpersonal & Organizacional & Proactividad \\
\cline { 2 - 6 } Orientación sexual & 0.264 & 0.239 & 0.45 & 0.438 & 0.406 \\
Estado civil & 0.067 & 0.089 & 0.396 & 0.447 & 0.973 \\
Grado de instrucción & 0.861 & 0.092 & 0.171 & 0.301 & 0.896 \\
Nombre de la empresa & 0.442 & 0.798 & 0.659 & 0.702 & 0.506 \\
Puesto & 0.243 & 0.534 & 0.265 & 0.195 & 0.362 \\
Ingreso salarial & 0.119 & 0.585 & 0.681 & 0.388 & 0.116 \\
Edad & 0.256 & 0.516 & 0.498 & 0.268 & 0.137 \\
Número de personas que & 0.064 & 0.058 & 0.792 & 0.669 & 0.932 \\
dependen & 0.307 & 0.365 & 0.791 & 0.869 & 0.98 \\
Área laboral & 0.732 & 0.973 & 0.349 & 0.243 & 0.326 \\
Tiempo de servicio & & & & & \\
\hline
\end{tabular}

\section{Interpretación de los datos o discusión}

El estudio de las relaciones interpersonales en trabajadores de medios de comunicación es en sumo necesario (Martínez, Matus y Villar, 2009), ya que su desempeño influye de forma social, en la calidad del trabajo que realizan los trabajadores operativos y administrativos (Jefes y subordinados) cuyo desempeño colige en la información que a través de la radio y la televisión se emiten todos los días en el que están involucrados de las noticias, los reportajes y demás información que editan, es así que se analiza en este estudio si el desempeño laboral se relaciona con las relaciones interpersonales afectivas en un grupo de trabajadores de radio y televisión en Lima Metropolitana.

Se encontró que existe una correlación estadísticamente significativa entre la dimensión interpersonal (de desempeño laboral) y la dimensión de relaciones con los pares (de relaciones afectivas) en trabajadores de radio y televisión de Lima Metropolitana, entendiendo que las personas que tienen un mayor desempeño en trabajar en equipo presentan una mejor relación con sus pares, así como señala De la Peña (2014).Se identificó relación significativa entre las dimensiones de relaciones entre pares y relaciones con el jefe, apoyando la hipótesis de que son parte del mismo constructo y que están asociadas entre sí, como señala Yañez et al. (2010).

Los resultados evidencian que existe una relación fuerte y positiva entre las dimensiones de la escala de desempeño laboral, esto concuerda con la hipótesis que plantean Coleman \& Borman (2000), asimismo con los planteamientos que sostiene Mayer, Salovey \& Caruso (2000) al diseñar la escala y conceptualizar el desempeño laboral en tres dimensiones (escrupulosidad, interpersonal y organizacional); asimismo, se reportan evidencias de confiabilidad y validez del 
"cuestionario de conductas de desempeño laboral", siendo adecuada su utilización en los trabajadores de radio y televisión de Lima Metropolitana, evaluados en el presente estudio. Lo que infiere que los resultados presentados son válidos y confiables.

Se evidencia que la escala de relaciones interpersonal en el trabajo presenta adecuadas propiedades métricas en trabajadores de radio y televisión de Lima Metropolitana, esto se relaciona con los estudios realizados por Yáñez (2006) y Yañez et al. $(2010,2009)$ de las propiedades psicométricas del instrumento. Esto apoyaría su uso en diferentes contextos. Por otra parte, se encuentra que las mujeres trabajadoras de radio y televisión en la muestra evaluada presentan un mayor nivel de relaciones con los pares que sus colegas hombres. Esto se relacionaría a los estudios de Goleman $(1996,1999)$ que hablan de diferentes habilidades sociales y cognitivas según el sexo. Asimismo, se puede interferir que las mujeres en promedio presentan mayores niveles de relaciones entre los pares que los hombres.

Existen diferencias significativas entre la relación con el jefe y la relación con los pares, según el número de hijos, si bien no se ha encontrado antecedentes de esta relación, si es necesario seguir estudiándolo para poder generar mayor evidencia de que a mayor número de hijos los participantes llegan a presentar un mayor nivel de relaciones con los pares y con los jefes. Se hallan diferencias entre los niveles de productividad según el tipo de contrato, sin embargo, no se reportan investigaciones en nuestro contexto que hayan encontrado una diferencia según la proactividad y el tipo de contrato, es así que es necesario seguir estudiando a mayor profundidad esta diferencia ya que podríamos inferir que personas con un tipo de contrato no fijo podrían ser más proactivas en búsqueda de que renueve su contrato, en comparación con personas con contrato fijo.

Finalmente si bien no existen diferencias en las relaciones interpersonales afectivas ni desempeño laboral en los trabajadores de radio y televisión de Lima Metropolitana, según el grado académico, la condición laboral, el tiempo de servicio, la edad, el estado civil, la carga familiar, el tipo de puesto, el nivel jerárquico, el área y el ingreso salarial, es necesario poder realizar nuevas investigaciones con una muestra más amplia que permitan identificar que no existe diferencias entre estas dos variables y las condiciones sociodemográficas. Es decir, el comportamiento de los participantes del estudio es homogéneo respecto de las relaciones interpersonales afectivas y el desempeño laboral.

\section{CONCLUSIONES}

Al finalizar el estudio, se concluye que en los trabajadores de radio y televisión de Lima Metropolitana evaluados existe una prevalencia del $41.1 \%$ en el nivel promedio de relaciones interpersonales afectivas en trabajadores de radio y televisión de Lima Metropolitana. Por otra parte, el 32.2\% reportó niveles bajos en 
relaciones interpersonales afectivas y solo el $26.7 \%$ en el nivel bueno. Además, se logró identificar que $38.9 \%$ de los trabajadores se encuentran el nivel promedio del desempeño laboral, el $28.9 \%$ reportó niveles bajos de desempeño laboral y un $32.2 \%$ en el nivel bueno.

Por otro lado, se concluye que no existe relación entre los puntajes totales de las relaciones interpersonales y el desempeño laboral en trabajadores de radio y televisión de Lima Metropolitana. Sin embargo, existe una correlación estadísticamente significativa entre la dimensión interpersonal (de desempeño laboral) y la dimensión relaciones con los pares (de relaciones afectivas) entendiendo que las personas que tienen un mayor desempeño y trabajan en equipo presentan una mejor relación con sus pares.

Asimismo, en relación al sexo, se identificó que las mujeres trabajadoras de radio y televisión de Lima Metropolitana presentan mejores relaciones con los pares que sus colegas hombres; y por otro lado, los varones muestran diferencias en el desempeño laboral en comparación con las mujeres, ya que ellos muestran niveles de desempeño más bajos que las mujeres.

Finalmente, no se observan diferencias significativas en las relaciones interpersonales afectivas ni desempeño laboral en los trabajadores de radio y televisión de Lima Metropolitana, según el grado académico, la condición laboral, el tiempo de servicio, la edad, el estado civil, la carga familiar, el tipo de puesto, el nivel jerárquico, el área de trabajo ni el ingreso salarial.

A la luz de los hallazgos y la discusión de los resultados se sugiere a las organizaciones desarrollar programas de capacitación centradas en la mejora de las habilidades interpersonales afectivas entre pares, y de esta manera generar la predisposición al trabajo en equipo. En este sentido, continuar desarrollando estudios sobre las relaciones interpersonales en la empresa y su influencia en el desempeño laboral, resulta sumamente relevante en esta área de la psicología organizacional. Asimismo, se recomienda también, que estudios posteriores enfaticen sobre el trabajo psicométrico en escalas que se ajusten a la realidad nacional de las organizaciones. Finalmente, se exhorta a los psicólogos del campo organizacional que continúen generando evidencia empírica de los comportamientos que tienen lugar en las organizaciones a fin de que las decisiones puedan resultar más sólidas para la empresa.

\section{REFERENCIAS}

Chiavenato,I. ( 2002). Gestión del Talento Humano. Bogotá:Mc.Graw-Hill

Chiaburu, D. \& Harrison, D. (2008). Do peers make the place? Conceptual synthesis and meta-analysis of coworker effects on perceptions, attitudes, OCBs, and performance. Journal of Applied Psychology, 93 (5), 1082-1103. 
Coleman, V. \& Borman, W. (2000). Investigating the underlying structure of the citizenship performance domain. Human resource management review, 10(1), 25-44.

Costello, A. \& Osborne, J. (2005). Best practices in exploratory factor analysis: Four recommendations for getting the most from your analysis. Practical Assessment Research \& Evaluation, 10(7), 1-9.

De la Peña, Y. (2014). Valores laborales y trabajo en equipo (estudio realizado en Construfácil Coatepeque). Tesis para optar el grado el grado de licenciatura en Psicóloga Industrial/Organizacional Universidad Rafael Landívar. Guatemala

De Vellis, R. F. (2012). Scale Development, Theory and Applicatons. Los Ángeles: SAGE.

Flores, M. y Rodríguez, C. (2006). Relación entre compromiso organizacional y desempeño contextual. Tesis para optar el grado el grado de licenciatura en Relaciones Industriales en la Universidad Católica Andrés Bello. Venezuela.

Goleman, D. (1996). Inteligencia emocional. Barcelona: Kairós.

Goleman, D. (1999). La práctica de la inteligencia emocional. Barcelona: Kairós.

Halbesleben, J. R. B., \& Wheeler, A. R. (2008). The relative roles of engagement and embeddedness in predicting job performance and intention to leave. Work \& Stress, 22(3), 242-256. http://dx.doi.org/10.1080/02678370802383962

Hernández. R., Fernández. C. y Baptista. P. (2010). Metodología de la Investigación (6 ${ }^{\mathrm{a}}$ ed.). México D.F.: McGraw-Hill.

Hogan, P. (2004). Psicometría. México: Manual Moderno

Juaneda, E. y González, M. (2007). Definición, antecedentes y consecuencias del compromiso organizativo. 3590-3609. Recuperado de https://ialnet.unirioja.es/ servlet/articulo?codigo $=2234965$

Lévy-Leboyer, C. (1997). Gestión de competencias. Barcelona, Gestión 2000

Mayer, J. D., Salovey, P., \& Caruso, D. R. (2000). Models of emotional intelligence. In R. J. Sternberg (Ed.), Handbook of intelligence (pp. 396 - 420). Cambridge, England: Cambridge University Press.

Muñiz, J. (2002). Teoría clásica de los test. Editorial Pirámide: Madrid.

Martínez, S., Matus, J. y Villar, H. (2009). La condición laboral del periodista en Chiapas. Revista Latina de Comunicación Social, 64, 444-460.

Montoya y Boyero (2015). El recurso humano como elemento fundamental para la gestión de calidad y la competitividad organizacional. Revista Científica Visión de Futuro, 20,2 , pp. $1-20$

Pohlmann, J. (2004). Use and interpretation of factor analysis in The Journal of Educational Research: 1992-2002. The Journal of Educational Research, 98(1), 14-22.

Sánchez, H., y Reyes, C. (2006). Metodología y diseños en la investigación científica. Lima: Editorial Visión Universitaria 
Swanson, R. A., \& Holton, E. F (2001). Foundations of Human Resource Development. San Franciso: Berrett-Koehler.

Yáñez, R. (2006). Los componentes de la confiabilidad en las relaciones interpersonales entre profesores universitarios. Estudios Pedagógicos, 12(1), 77-90.

Yáñez, R., Loyola, G. \& Huenumilla, F. (2009). La confiabilidad en el líder. Un estudio sobre las enfermeras jefe de un hospital. Ciencia y Enfermería, 15(3), 77-89.

Yañez, R., Arenas, M. y Ripoll, M. (2010). El impacto del desempeño laboral en la satisfacción laboral general. Liberabit, 16(2), 193-202. 